\title{
EIGENVALUES OF COMPLEX TRIDIAGONAL MATRICES
}

\author{
by P. M. GIBSON $\dagger$ \\ (Received 4th September 1970)
}

Results of Arscott (1) and Jayne (3) on real matrices are generalized to obtain bounds for the real parts of the eigenvalues of certain complex tridiagonal matrices, and bounds for the imaginary parts of the eigenvalues of other tridiagonal matrices are given. It is shown that analogous results hold for zeros of the permanent of certain characteristic matrices.

If $A$ is an $n$-square matrix and $0 \leqq j<k \leqq n$, let $A(j, k]$ denote the $(k-j)$ square principal submatrix lying in rows (and columns) $j+1, \ldots, k$ of $A$. Let $\Omega$ be a complex $n$-square tridiagonal matrix,

$$
\Omega=\left[\begin{array}{llllll}
b_{1} & c_{1} & 0 & \ldots 0 & 0 \\
a_{1} & b_{2} & c_{2} & \ldots 0 & 0 \\
0 & a_{2} & b_{3} & \ldots 0 & 0 \\
\vdots & \vdots & \vdots & \vdots & \vdots \\
0 & 0 & 0 & \ldots & b_{n-1} & c_{n-1} \\
0 & 0 & 0 & \ldots & a_{n-1} & b_{n}
\end{array}\right],
$$

where $a_{k} c_{k}$ is real, $k=1, \ldots, n-1$.

Lemma. If $b_{j}=0$ for $j=1, \ldots, n$, and $a_{k} c_{k}<0$ for $k=1, \ldots, n-1$, then there exists an $n$-square non-singular diagonal matrix $D=\left[d_{j}\right]$ with $d_{1}=1$ such that $D^{-1} \Omega D$ is skew-hermitian.

Proof. The proof is inductive. Clearly the lemma is true for $n=1$. Suppose that $n \geqq 2$. Define the $n$-square diagonal matrix $P=\left[p_{j}\right]$ by letting

If

$$
p_{2}=\sqrt{\left|a_{1} / c_{1}\right|}, \quad p_{j}=1 \text { if } j \neq 2 \text {. }
$$

$$
\Gamma=\left[\gamma_{j k}\right]=P^{-1} \Omega P
$$

then

$$
\gamma_{1 j}=-\bar{\gamma}_{j 1}, \quad j=1, \ldots, n,
$$

and the $(n-1)$-square tridiagonal matrix $\Gamma(1, n]$ satisfies the hypotheses of the lemma. By the inductive assumption there exists an $(n-1)$-square non-singular

$\dagger$ This research was sponsored by the National Science Foundation through a grant by the Research Committee of the University of Alabama in Huntsville. 
diagonal matrix $Q=\left[q_{j}\right]$ with $q_{j}=1$ such that $Q^{-1} \Gamma(1, n] Q$ is skew-hermitian. Let

$$
R=\left[\begin{array}{ll}
1 & 0 \\
0 & Q
\end{array}\right], \quad D=P R
$$

Then $D=\left[d_{j}\right]$ is an $n$-square non-singular diagonal matrix with $d_{1}=1$, and $D^{-1} \Omega D$ is skew-hermitian.

Theorem 1. If $\lambda$ is an eigenvalue of $\Omega$ and $a_{k} c_{k} \leqq 0$ for $k=1, \ldots, n-1$, then

$$
\min \left\{\operatorname{Re}\left(b_{j}\right) \mid j=1, \ldots, n\right\} \leqq \operatorname{Re}(\hat{\lambda}) \leqq \max \left\{\operatorname{Re}\left(b_{j}\right) \mid j=1, \ldots, n\right\} .
$$

Proof. First suppose that $a_{k} c_{k}<0$ for $k=1, \ldots, n-1$. Let $B$ be the $n$ square diagonal matrix with diagonal equal to the diagonal of $\Omega$. According to the lemma, there is an $n$-square non-singular diagonal matrix $D$ and a skewhermitian matrix $C$ such that

$$
D^{-1}(\Omega-B) D=C .
$$

Then $\lambda$ is an eigenvalue of $B+C$, and

$$
B+C+(B+C)^{*}=B+\bar{B}
$$

Hence, if $\mu$ and $v$ are, respectively, the smallest and the largest eigenvalues of $(B+\bar{B}) / 2$ then $(4$, p. 142)

$$
\mu \leqq \operatorname{Re}(\lambda) \leqq v .
$$

However,

$$
\begin{aligned}
& \min \left\{\operatorname{Re}\left(b_{j}\right) \mid j=1, \ldots, n\right\}=\mu, \\
& v=\max \left\{\operatorname{Re}\left(b_{j}\right) \mid j=1, \ldots, n\right\} .
\end{aligned}
$$

Hence, (1) holds, if $a_{k} c_{k}<0$ for $k=1, \ldots, n-1$. Now suppose that

$k=1, \ldots, n-1$. Let

$$
\begin{aligned}
& 1 \leqq k_{1}<k_{2}<\ldots<k_{m} \leqq n-1, \\
& a_{k} c_{k}=0 \text { if } k \in\left\{k_{1}, \ldots, k_{m}\right\}, \\
& a_{k} c_{k}<0 \text { if } k \notin\left\{k_{1}, \ldots, k_{m}\right\},
\end{aligned}
$$

$$
k_{0}=0, \quad k_{m+1}=n \text {. }
$$

We have

$$
\operatorname{det}(z I-\Omega)=\prod_{\alpha=0}^{m} \operatorname{det}\left(z I-\Omega\left(k_{\alpha}, k_{\alpha+1}\right]\right) \text {. }
$$

Hence, since $\lambda$ is an eigenvalue of $\Omega$, it is an eigenvalue of some $\Omega\left(k_{\alpha}, k_{\alpha+1}\right]$. Therefore, by the first part of the proof, $\min \left\{\operatorname{Re}\left(b_{j}\right) \mid j=k_{\alpha}+1, \ldots, k_{\alpha+1}\right\} \leqq \operatorname{Re}(\lambda) \leqq \max \left\{\operatorname{Re}\left(b_{j}\right) \mid j=k_{\alpha}+1, \ldots, k_{\alpha+1}\right\}$. Hence, (1) is true. 
Arscott (1) proved that (1) holds if $\lambda$ is real, $\Omega$ is real, and $a_{k} c_{k}<0$ for $k=1, \ldots, n-1$. Jayne (3) generalized Arscott's result by removing the requirement that $\lambda$ be real. Our method of proving Theorem 1 is quite different from the techniques used by Arscott and Jayne. then

Theorem 2. If $\lambda$ is an eigenvalue of $\Omega$ and $a_{k} c_{k} \geqq 0$ for $k=1, \ldots, n-1$,

$$
\min \left\{\operatorname{Im}\left(b_{j}\right) \mid j=1, \ldots, n\right\} \leqq \operatorname{Im}(\lambda) \leqq \max \left\{\operatorname{Im}\left(b_{j}\right) \mid j=1, \ldots, n\right\} .
$$

Proof. Apply Theorem 1 to the tridiagonal matrix $-i \Omega$.

Theorem 3. If $\lambda$ is a zero of $\operatorname{per}(z I-\Omega)$ and $a_{k} c_{k} \geqq 0$ for $k=1, \ldots, n-1$, then (1) is satisfied.

Proof. It follows from (2) that if $\Gamma$ is the tridiagonal matrix obtained from $\Omega$ by replacing each $c_{k}$ by $-c_{k}$ then

$$
\operatorname{per}(z I-\Omega)=\operatorname{det}(z I-\Gamma) \text {. }
$$

Hence, since $\lambda$ is a zero of $\operatorname{per}(z I-\Omega), \lambda$ is an eigenvalue of $\Gamma$. Therefore, by Theorem 1, (1) holds.

Similarly it can be shown that Theorem 2 implies the following.

Theorem 4. If $\lambda$ is a zero of $\operatorname{per}(z I-\Omega)$ and $a_{k} c_{k} \leqq 0$ for $k=1, \ldots, n-1$, then (2) is satisfied.

\section{REFERENCES}

(1) F. M. ARscotT, Latent roots of tri-diagonal matrices, Edinburgh Math. Notes 44, 5-7 [in Proc. Edinburgh Math. Soc. (2) 12 (1961)].

(2) P. M. Gibson, An identity between permanents and determinants, Amer. Math. Monthly 76 (1969), 270-271.

(3) J. W. JAYNE, An exclusion theorem for tri-diagonal matrices, Proc. Edinburgh Math. Soc. (2) 16 (1969), 251-253.

(4) M. MARcus and H. MINc, A Survey of Matrix Theory and Matrix Inequalities (Allyn and Bacon, Boston, 1964).

University of Alabama in Huntsville

Huntsville, Alabama 35807

U.S.A. 